\title{
Feeding ecology of a nesting population of the Common Buzzard (Buteo buteo) in the Upper Nitra Region, Central Slovakia
}

\section{Potravná ekológia hniezdnej populácie myšiaka hôrneho (Buteo buteo) na hornom Ponitrí, stredné Slovensko}

\author{
Karol ŠOTNÁR \& Ján OBUCH
}

\section{Šotnár K \& Obuch J 2009: Feeding ecology of a nesting population of the Common Buzzard (Buteo buteo) in the Upper Nitra region, Central Slovakia. Slovak Rapt J 3: 13-20.}

\begin{abstract}
During routine checks of the nests of the Common Buzzard in the Upper Nitra region (Central Slovakia) we collected food remains and recorded all prey given to the nestlings. We present results from the period 2006-2008. 606 food items were determined, comprising mainly mammals (67\%). Birds were also frequent (17\%), less so amphibians and reptiles (7\%) and invertebrates (10\%). During the rodent population peak in 2007, Buzzard pairs nesting in the valley preyed mainly on Microtus arvalis, whereas pairs living at the rim of the valley fed on Myodes glareolus and Talpa europaea, and higher in the Vtáčnik Mts birds fed on Apodemus flavicollis. After a massive decline in the rodent population in the following year 2008, the survival rate of the nestlings was very low and the proportion of invertebrates in their diet increased. Birds that were most frequently preyed upon included juvenile Turdus philomelos and Garrulus glandarius, prey identified from amphibian was mainly Bufo bufo, and from reptile prey was largely Anguis fragilis.
\end{abstract}

\begin{abstract}
Abstrakt: Pri kontrolách hniezd myšiaka hôrneho v oblasti horného Ponitria (stredné Slovensko) sme zbierali zvyšky jeho potravy a zaznamenávali korist', prinesenú mlád’atám. Prezentujeme výsledky z rokov 2006-2008. Determinovali sme 606 kusov potravy, v ktorej dominujú cicavce (67\%). Početné sú aj vtáky (17\%), obojživelníky a plazy (7\%) a bezstavovce (10 \%). Pri vysokej populačnej hladine hlodavcov v roku 2007 bol u párov, hniezdiacich v kotline, dominantnou korist'ou druh Microtus arvalis, na okraji kotliny druhy Myodes glareolus a Talpa europaea a u párov, hniezdiacich vyššie v pohorí Vtáčnik druh Apodemus flavicollis. Po silnom poklese populačnej hladiny hlodavcov v nasledujúcom roku 2008 bola nízka úspešnost' hniezdenia myšiakov a v ich potrave stúpol podiel bezstavovcov. Z vtákov boli najčastejšie lovené mlád'atá druhov Turdus philomelos a Garrulus glandarius, z obojživelníkov Bufo bufo a z plazov Anguis fragilis.
\end{abstract}

Key words: Buteo buteo, diet, Upper Nitra region, Central Slovakia

Karol Šotnár, Gavloviča 1/5, SK-971 01 Prievidza, Slovakia. E-mail: karol_sotnar@post.sk.

Ján Obuch, Comenius University, Botanical Garden, SK-038 15 Blatnica, Slovakia. E-mail: obuch@rec.uniba.sk.

Acknowledgments: We owe our gratitude to Martin Dúbrava and Branislav Beniska who physically examined the Buzzard nests, collected pellets and food remains of the Common Buzzard in the Upper Nitra region.

\section{Introduction}

In contrast to owls, bones are digested to a greater degree in the pellets of birds of prey. Therefore the investigation of their diet requires employing several different methods. During the nesting period, examination of pellets, determination of bone and feather remains in the nest can be used, as well as recording prey items presented to the nestlings. Prey remains in the nests of the Buteo buteo were evaluated by Šotnár $(2003,2007)$. In South Moravia this method was used for examining the food of the B. buteo by Voříšek et al. (1997) and Horák \& Matušík (2000), a smaller sample from the Czech Republic and
Moravia was examined by Toufar (1958). In Germany, the same method was used to examine the food of $B$. buteo by Wuttky (1963) and Mebs (1964).

In the past when birds of prey were hunted legally and shot individuals were concentrated in taxidermist workshops, the stomach content of the birds could be examined. Sládek $(1961,1966)$ used this method in cooperation with the taxidermist workshop in Žiar nad Hronom. In the same manner, Sabadoš \& Sládek (1968) studied the diet of the birds of prey shot in the pheasant reserve during an exceptionally harsh winter. Ševčík (1980) examined the food of the Buzzards shot in the pheasant reserves in the 
Czech Republic. Extensive material collected by Rörig from Germany was studied by Uttendörfer (1939).

Comprehensive data from Central Europe is summarized in the papers of Glutz et al. (1989) and Cramp \& Simmons (1980). Our aim was to evaluate and compare the diet of the Common Buzzard population in three nesting seasons and three elevation zones of the Upper Nitra region.

\section{Material and Methods}

In 2006-2008 we collected pellets and recorded food remains in the nests of the Common Buzzard in the Upper Nitra region. Nests were examined in May-July. Our work builds upon the study by Šotnár \& Topercer (2009). Collected material was classified into two cathegories:

Provisions - visual examination of food items. Determination of whole individuals, food remains, feather and fur directly in the nest.

Pellets - analysis of pellets and bone remains. All samples were processed in $5 \% \mathrm{NaOH}$ solution to dissolve the feather and hair material. Bone fragments were used for the determination of vertebrate prey and elytrae and jaws were used to determine invertebrates. Mammals were determined according to cranial fragments, moles were also identified using humerus, Microtids using the lower molar teeth $\mathrm{M}_{1}$. Birds were determined according to 5 types of bone fragments: beaks, humerus, metatarsus and tarsometatarsus. Reptiles were determined according to jaws, skeletons and skin or scale remains. Food samples collected in 2007 and 2008 were classified into 3 zones:

A. Nesting pairs from the valley, coline zone $300-500$ $\mathrm{m}$ a. s. 1. (agrocenoses, field forests, riparian vegetation, 42 samples);

B. Nesting pairs from the piedmont area of Vtáčnik Mts, submontane zone 500-700 $\mathrm{m}$ a. s. 1. (forest edge up to $1 \mathrm{~km}$ deep into the forest, 36 samples);

C. Nesting pairs from the mountain range Vtáčnik Mts., montane zone $700-1100 \mathrm{~m}$ a. s. 1. (deep in the forests, approximately $2-5 \mathrm{~km}$ from the rim of the valley, 15 samples).

To evaluate the results in the Table 1-4 the method of marked differences from the mean (MDFM; Obuch 2001) was used. In the tables samples are sorted according to their similarity. Species in the upper part of the table are sorted that those with marked positive deviations are arranged in blocks bordered by solid lines. Species with less marked deviations are sorted under the dashed line according to descending frequency. Those with frequency less than 5 are shown in Table 1. The lower part of the table shows sums for individual vertebrate and invertebrate classes. The diversity index $\mathrm{H}^{6}$ is calculated according to the formula of Shannon \& Weaver (1949).

\section{Results}

In 2006-2008 we studied food remains in the nests of $B$. buteo and determined 606 prey items. The most dominant part of the diet comprised mammals (Mammalia, 67\%), less frequent were birds (Aves, 17\%), amphibians and

Tab. 1. Food composition of B. buteo in the Upper Nitra Valley, inter-annual comparison

Tab. 1. Potrava B. buteo na hornej Nitre, porovnanie rokov

\begin{tabular}{|c|c|c|c|c|c|c|}
\hline $\begin{array}{l}\text { year (no.) / rok (č.) } \\
\text { species / druhy }\end{array}$ & $\begin{array}{c}2006 \\
(1)\end{array}$ & & $\begin{array}{l}007 \\
(2)\end{array}$ & $\begin{array}{c}2008 \\
(3)\end{array}$ & $\Sigma$ & $\%$ \\
\hline Apodemus flavicollis & $1-1$ & $1+$ & 351 & $1-\quad 0$ & 36 & 5.94 \\
\hline Coleoptera sp. & 5 & & 201 & $1+19$ & 44 & 7.26 \\
\hline Hymenoptera sp. & & & 11 & $1+7$ & 8 & 1.32 \\
\hline Microtus arvalis & 38 & & 1101 & $1-13$ & 161 & 26.57 \\
\hline $\bar{T}$ Talpa europaea & 27 & & 65 & 21 & 113 & $18 . \overline{65}$ \\
\hline Myodes glareolus & 6 & & 37 & 9 & 52 & 8.58 \\
\hline Turdus philomelos & 5 & & 11 & 1 & 17 & 2.81 \\
\hline Garrulus glandarius & 5 & & 6 & 5 & 16 & 2.64 \\
\hline Sorex araneus & & & 10 & 3 & 13 & 2.15 \\
\hline Bufo bufo & 1 & & 9 & 1 & 11 & 1.82 \\
\hline Anguis fragilis & 3 & & 2 & 3 & 8 & 1.32 \\
\hline Coccothraustes coccothr. & 4 & & 2 & 2 & 8 & 1.32 \\
\hline Sturnus vulgaris & 1 & & 5 & 1 & 7 & 1.16 \\
\hline Arvicola terrestris & 2 & & 4 & 1 & 7 & 1.16 \\
\hline Buteo buteo & & & 2 & 4 & 6 & 0.99 \\
\hline Columba livia domestica & 1 & & 2 & 2 & 5 & 0.83 \\
\hline Terricola subterraneus & 2 & & 3 & & 5 & 0.83 \\
\hline Mammalia & 78 & & 275 & 1- 53 & 406 & 67.00 \\
\hline Aves & 26 & & 54 & 21 & 101 & 16.67 \\
\hline Amphibia, Reptilia & 13 & & 23 & 5 & 41 & 6.77 \\
\hline Evertebrata & 8 & 1- & & $1+26$ & 58 & 9.57 \\
\hline$\Sigma$ & 125 & & 376 & 105 & \multicolumn{2}{|c|}{606100.00} \\
\hline $\begin{array}{l}\text { H' diversity index / } \\
\text { index diverzity }\end{array}$ & 2.49 & & 2.58 & 2.67 & \multicolumn{2}{|l|}{2.74} \\
\hline
\end{tabular}

Other species / Ostatné druhy (year no. / rok č. - number I počet): Erinaceus concolor (1-1; 3-1), Sorex minutus (1-1), Neomys fodiens (2-1; 3-1), Crocidura suaveolens (2-1), Lepus europaeus (2-3; $3-1)$, Sciurus vulgaris (3-2), Mus cf. musculus (2-1), Apodemus sylvaticus (2-1), Microtus agrestis (2-1), Mustela nivalis (2-1), Artiodactyla sp. (2-2; 3-1), Columba oenas (3-1), Columba palumbus (1-1; 2-1; 3-1), Columba sp. (2-2), Aegolius funereus (2-1), Strix aluco (1-1), Dryocopus martius (2-1), Dendrocopos medius (2-1), Dendrocopos sp. (2-4; 3-1), Anthus sp. (2-1), Locustella fluviatilis (1-1), Turdus sp. (2-1), Sitta europaea (2-2), Emberiza citrinella (3-1), Fringilla coelebs (1-1; 3-1), Pica pica (2-1), Passeriformes sp. (1-6; 2-11; 3-1), Bombina variegata (1-1), Rana temporaria (1-1; 2-1), Rana cf. esculenta (2-1), Amphibia sp. (1-3), Lacerta agilis (2-1; 3-1), Natrix natrix (2-2), Elaphe longissima (1-1; 2-4), Coronella austriaca (1-1), Colubridae sp. (1-2; 2-3), Orthoptera sp. (1-3; 2-1), Melolontha melolontha (2-1), Diptera sp. (2-1) 
reptiles (Amphibia and Reptilia, 7\%; Fig. 1-7). Invertebrates (Evertebrata, 10\%) are numerous, but comprise an insignificant part of the total biomass.

The most dominant prey species Microtus arvalis $(26.6 \%)$ was less frequent in 2008, Talpa europaea $(18.7 \%)$ is equally frequent in samples from 2006, 2007 and 2008, Apodemus flavicollis (5.9\%) is more frequent in 2007. The lack of rodents in 2008 is partially compensated by increased proportion of invertebrates, including mainly insects of the orders Coleoptera and Hymenoptera (Table 1). The most frequent mammals include Myodes glareolus $(8.6 \%)$ and Sorex araneus (2.2\%). From birds B. buteo fed most frequently on juveniles of Turdus philomelos (2.8\%) and Garrulus glandarius (2.6\%). Bufo bufo (1.8\%) was among the most frequent amphibian prey and Anguis fragilis $(1.3 \%)$ was the most frequent reptile .

Tab. 2. Food composition of B. buteo in the Upper Nitra Valley, comparison between sampling methods

Tab. 2. Potrava B. buteo na hornej Nitre, porovnanie metód zberov

\begin{tabular}{|c|c|c|c|c|}
\hline $\begin{array}{l}\text { species / druhy } \\
\text { sample type / druh zberu }\end{array}$ & $\mathbf{a}$ & b & $\Sigma$ & $\%$ \\
\hline Buteo buteo & $1-$ & $1+6$ & 6 & 0.99 \\
\hline Turdus philomelos & $1-$ & $1+13$ & 17 & 2.81 \\
\hline Coccothraustes coccothr. & $1-$ & $1+$ & 8 & 1.32 \\
\hline Sturnus vulgaris & $1-$ & $1+$ & 7 & 1.16 \\
\hline Garrulus glandarius & $2-$ & $1+$ & 16 & 2.64 \\
\hline Passeriformes sp. & $2-$ & $2+$ & 18 & 2.97 \\
\hline Apodemus flavicollis & 36 & $2-$ & 36 & 5.94 \\
\hline Myodes glareolus & 52 & 2- & 52 & 8.58 \\
\hline Microtus arvalis & 144 & 17 & 161 & 26.57 \\
\hline Coleoptera sp. & 42 & $1-$ & 44 & 7.26 \\
\hline $\bar{T}$ Talpa europaea & 92 & 21 & $11 \overline{3}$ & $18 . \overline{65}$ \\
\hline Sorex araneus & 13 & & 13 & 2.15 \\
\hline Bufo bufo & 11 & & 11 & 1.82 \\
\hline Anguis fragilis & 7 & 1 & 8 & 1.32 \\
\hline Hymenoptera sp. & 8 & & 8 & 1.32 \\
\hline Arvicola terrestris & 7 & & 7 & 1.16 \\
\hline Terricola subterraneus & 5 & & 5 & 0.83 \\
\hline Columba livia dom. & 5 & & 5 & 0.83 \\
\hline Elaphe longissima & 3 & 2 & 5 & 0.83 \\
\hline Mammalia & 363 & 43 & 406 & 67.00 \\
\hline Aves & $2-17$ & 84 & 101 & 16.67 \\
\hline Amphibia, Reptilia & 32 & 9 & 41 & 6.77 \\
\hline Evertebrata & 55 & 1- & 58 & 9.57 \\
\hline $\bar{\Sigma}$ & 467 & 139 & 606 & 100.00 \\
\hline $\begin{array}{l}\mathrm{H}^{\prime} \text { diversity index / } \\
\text { index diverzity }\end{array}$ & 2.35 & 2.81 & 2.74 & \\
\hline
\end{tabular}

The pellets revealed three times more prey items than food provisions in the nests. Therefore the comparison of species composition using the MDFM method (Table 2) showed marked differences only in the less frequent material collected in the nests. The pellets comprised mainly mammals (77.7\%), including $M$. arvalis, $M$. glareolus and $A$. flavicollis. Coleopterans were the most frequent invertebrate prey. Nest provisions included mainly birds (Aves, 60.4\%), namely G. glandarius, T. philomelos, Sturnus vulgaris and Coccothraustes coccothraustes.

The evaluation of $B$. buteo diet composition in relation to habitat was strongly influenced by the gradation of rodents in 2007 and the subsequent decline combined with unfavourable weather conditions in 2008. Great abundance of food in 2007 was reflected in the higher nesting success. Therefore in 2007 we collected a three times larger sample of food than in 2008. In 2007 the most frequent prey item of $B$. buteo in the valley included M. arvalis $(51.4 \%)$, in the piedmont of Vtáčnik, M. glareolus and T. europaea were more frequent in the montane zone, A. flavicollis was dominant. In 2008, Aves and Evertabrata were more frequent in the valley and T. europaea and Evertebrata in the piedmont area. The montane zone yielded only a small sample of 22 food items. Notable prey items included Aegolius funereus, Strix aluco and Locustella fluviatilis.

\section{Discussion}

Our data on the food composition of B. buteo from the Upper Nitra valley, which were collected through the analysis of pellets and food provisions in the nests, corresponds with the results of other authors using similar methods in South Bohemia and Germany. Since the works of Voříšek et al. (1997) and Horák \& Matušík (2000) only include prey determined to higher taxonomic levels, we decided to make comparison in Table 4 only with our own data from the determination of pellets at Pálava. All four studies on the diet of B. buteo, used in the comparison, show a lower proportion of the dominant species $M$. arvalis and higher proportion of the subdominant $T$. europaea. Birds can be seen in higher proportion, especially G. glandarius and T. philomelos species. In contrast to other studies, our results show higher proportion of S. araneus, A.flavicollis, C. coccothraustes and Elaphe longissima. In our results we also included findings of dead $B$. buteo nestlings, killed mainly as a result of cainism during the shortage of food in 2008. Studies using similar methods found other species more abundant such as Apodemus sylvaticus, Arvicola terrestris, Lacerta agilis, A. fragilis (Mebs 1964) Lepus europaeus, Alauda arvensis (Mebs 1964, Wuttky 1963), Cricetus cricetus, 


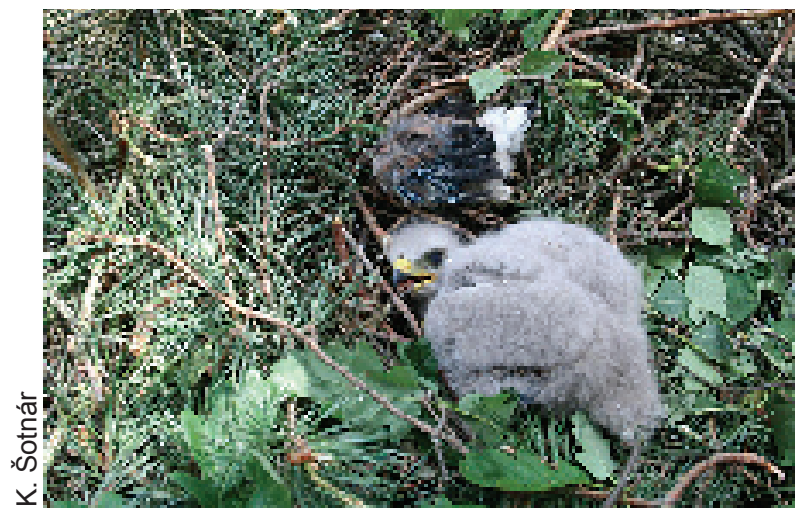

Fig. 1. The most frequently preyed bird - Garrulus glandarius (Vel'ká Čausa, 29 May 2008, 1 pull. 13-day and 1 egg).

Obr. 1. Najčastejšie lovený druh vtákov bol Garrulus glandarius (Vel'ká Čausa, 29. máj 2008, 1 pull. 13-dňové a 1 vajce).

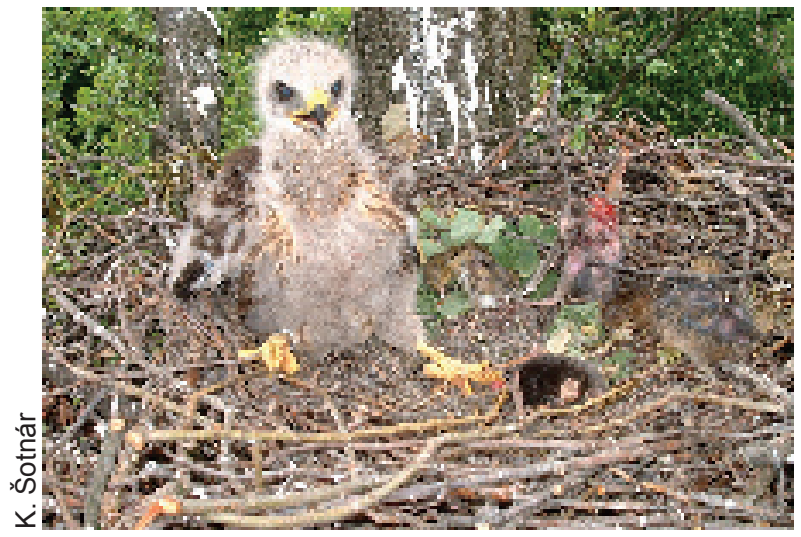

Fig. 3. Food supply - T. europaea, A. terrestris, L. fluviatilis, $T$. merula (Lehota pod Vtáčnikom, 6 June 2006, pull. 21 days old). Obr. 3. Zásoba potravy - T. europaea, A. terrestris, L. fluviatilis, $T$. merula (Lehota pod Vtáčnikom, 6. jún 2006, mlád’a 21-dňové).

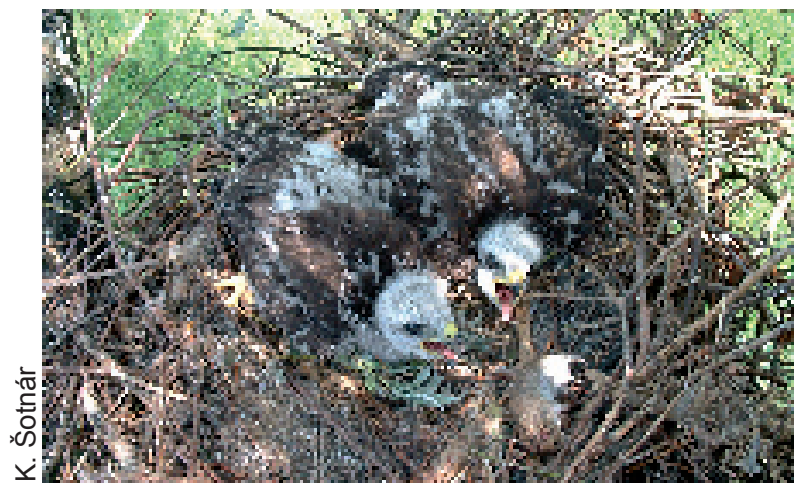

Fig. 5. Main food component of the Common Buzard - Microtus arvalis (Nováky, 6 June 2007, 2 pull. ca 28 days old).

Obr. 5. Hlavná zložka potravy myšiakov - Microtus arvalis (Nováky, 6. jún 2007, 2 pull. asi 28-dňové).

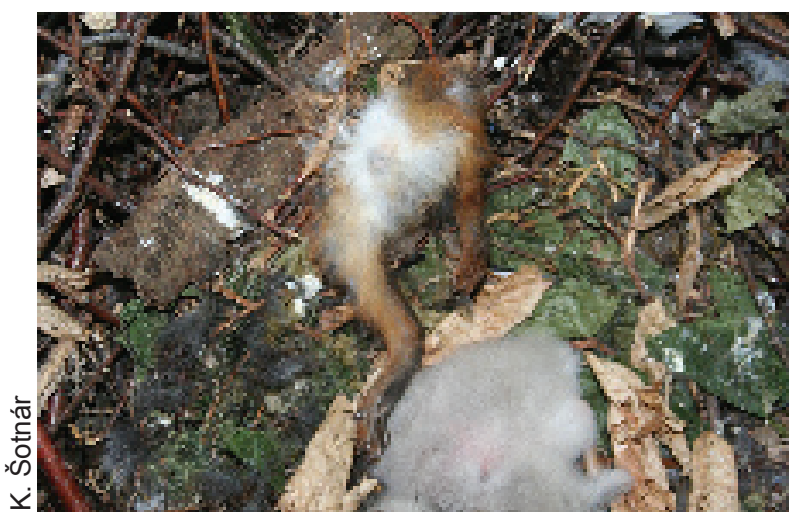

Fig. 2. Prey item in the nest - Sciurus vulgaris without head (Fančová, 27 May 2008, 2 pull. 5-7 days old).

Obr. 2. Potrava na hniezde - Sciurus vulgaris bez hlavy (Fančová, 27. máj 2008, 2 pull. 5-7-dňové).

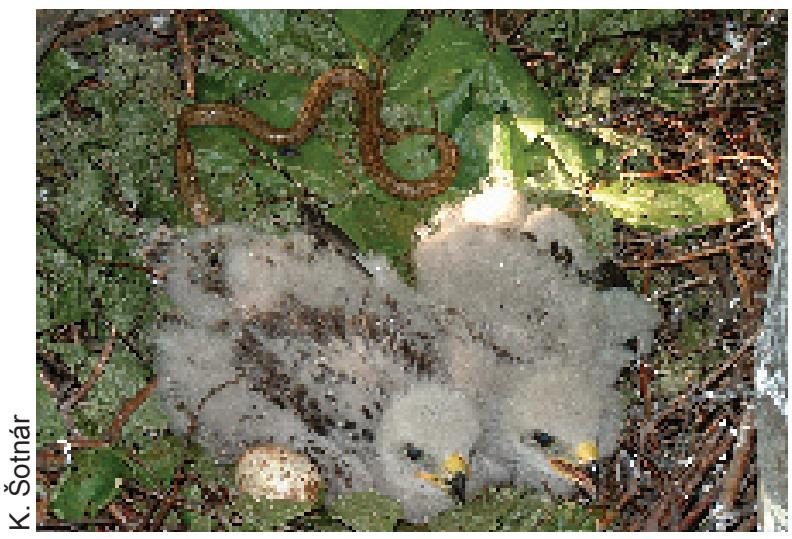

Fig. 4. Prey item - Coronella austriaca (Cígel', Vtáčnik, 24 May 2004, 2 pull. ca 18-20 days old and 1 egg).

Obr. 4. Potrava - Coronella austriaca (Cígel', Vtáčnik, 24. máj 2004, 2 pull. asi 18-20 dňové a 1 vajce).

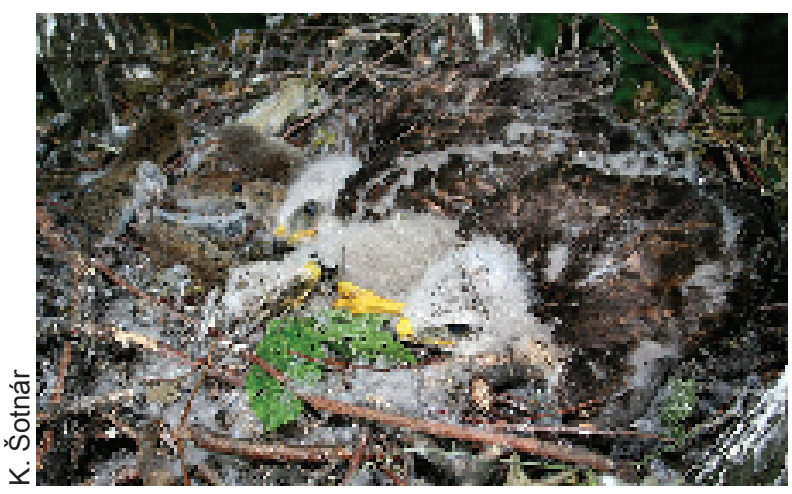

Fig. 6. Three nestlings in the nest, food supply $-6 \mathrm{M}$. arvalis (Kamenec p. Vtáčnikom, 24 May 2008).

Obr. 6. Tri mlád’atá na hniezde, potrava na hniezde $-6 \mathrm{M}$. arvalis (Kamenec p. Vtáčnikom, 24. máj 2008). 
Slovak Rapt J 2009, 3: 13-20. DOI: 10.2478/v10262-012-0028-0.

(C) Raptor Protection of Slovakia (RPS)

Tab. 3. Food composition of $B$. buteo in the Upper Nitra Valley, evaluation of 2007 and 2008 samples according to habitat zones Tab. 3. Potrava B. buteo na hornej Nitre, vyhodnotenie zberov z rokov 2007 a 2008 podla zón

\begin{tabular}{|c|c|c|c|c|c|c|c|c|c|c|}
\hline \multirow{3}{*}{$\begin{array}{l}\text { year / rok } \\
\text { zone / zóna // } \\
\text { species / druhy } \\
\text { Microtus arvalis }\end{array}$} & \multicolumn{3}{|c|}{2007} & \multicolumn{5}{|c|}{2008} & \multirow[b]{2}{*}{$\Sigma$} & \multirow[b]{2}{*}{$\%$} \\
\hline & A & B & C & \multicolumn{2}{|r|}{ A } & \multicolumn{2}{|c|}{ B } & C & & \\
\hline & $1+73$ & 34 & $1-$ & & 10 & $1-$ & 2 & $1-$ & 123 & 25.47 \\
\hline Myodes glareolus & $2-$ & 26 & 9 & & & & 3 & 6 & 46 & 9.52 \\
\hline Talpa europaea & 13 & $1+44$ & 8 & $1-$ & 1 & $1+$ & 18 & 2 & 86 & 17.81 \\
\hline Apodemus flavicollis & $1-$ & 15 & $1+15$ & & & & & & 35 & 7.25 \\
\hline Coleoptera sp. & 10 & $1-$ & 4 & $1+$ & 8 & & 7 & 4 & 39 & 8.07 \\
\hline Hymenoptera sp. & 1 & & & & 2 & $1+$ & 5 & & 8 & 1.66 \\
\hline$\overline{\text { Sorex araneus }}$ & 4 & 3 & 3 & & & & 1 & 2 & 13 & $2 . \overline{69}$ \\
\hline Turdus philomelos & 2 & 7 & 2 & & & & 1 & & 12 & 2.48 \\
\hline Garrulus glandarius & 3 & 3 & & & 3 & & 1 & 1 & 11 & 2.28 \\
\hline Bufo bufo & 2 & 5 & 2 & & & & 1 & & 10 & 2.07 \\
\hline Buteo buteo & & 2 & & & 4 & & & & 6 & 1.24 \\
\hline Sturnus vulgaris & 3 & 2 & & & 1 & & & & 6 & 1.24 \\
\hline Arvicola terrestris & 3 & 1 & & & 1 & & & & 5 & 1.04 \\
\hline Anguis fragilis & 2 & & & & 1 & & 1 & 1 & 5 & 1.04 \\
\hline Mammalia & 106 & 129 & 40 & $1-$ & 14 & & 25 & 14 & 328 & 67.91 \\
\hline Aves & 17 & 31 & 7 & $1+$ & 15 & & 4 & 3 & 77 & 15.94 \\
\hline Amphibia, Reptilia & 7 & 11 & 5 & & 1 & & 3 & 1 & 28 & 5.80 \\
\hline Evertebrata & 12 & 1- & 4 & $1+$ & 10 & $1+$ & 12 & 4 & 50 & 10.35 \\
\hline$\sum$ & 142 & 179 & 56 & & 40 & & 44 & 22 & 483 & 100.00 \\
\hline $\begin{array}{l}\mathrm{H}^{\prime} \text { diversity index / } \\
\text { index diverzity }\end{array}$ & 2.11 & 2.50 & 2.36 & & 2.44 & & 2.00 & 2.22 & 2.73 & \\
\hline
\end{tabular}

Zone: A - coline zone 300-500 m a. s. I., B - submontane zone 500-700 m a. s. I., C - montane zone 700-1100 m a. s. I. Zóna: A - kolínna 300-500 m n. m., B - submontánna 500-700 m n. m., C - montánna 700-1100 m n. m.

Emberiza citrinella, Columba livia domestica (Wuttky 1963) and Coleoptera (Voříšek et al. 1997; Table 4).

The papers focusing on the examination of stomach contents more frequently studied winter samples, dominated mainly by $M$. arvalis. Stomach contents of Common Buzzards shot in the pheasant reserves more often included Phasianus colchicus and Gallus gallus domestica species (Ševčík 1980). Invertebrates are usually better preserved in stomach samples than vertebrates, therefore Coleoptera, Orthoptera (Sládek 1961), as well as Lepidopteran larvae (caterpillars) were often found in the stomach contents (Ševčík 1980, Uttendörfer 1939).

According to our study from the Upper Nitra, small game (hare, pheasant, grey partridge) comprises less than $1 \%$ of the Common Buzzard's diet. Several authors (Sládek 1966, Horák \& Matušík 2000) suggested that small game were not originally preyed upon by $B$. buteo and that it only fed on their carcasses.

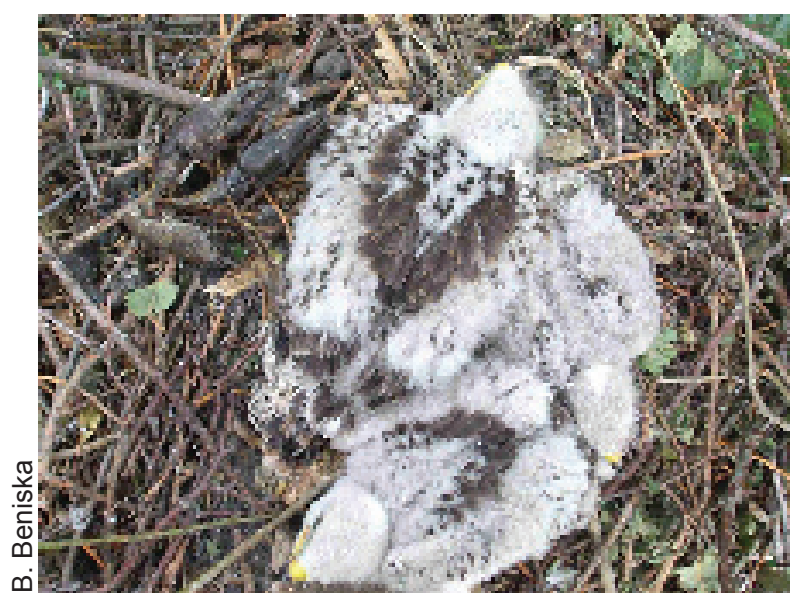

Fig. 7. Astacus sp. - unusual prey of Common Buzard. (Lehota pod Vtáčnikom, 30 May 2009).

Obr. 7. Astacus sp. - nezvyčajná potrava na hniezde myšiaka hôrneho. (Lehota pod Vtáčnikom, 30. máj 2009). 
Tab. 4. Evaluation of data on $B$. buteo diet in the Central Europe

Tab. 4. Vyhodnotenie údajov o potrave $B$. buteo v strednej Európe

\begin{tabular}{|c|c|c|c|c|c|c|c|c|c|c|c|c|c|}
\hline species / druhy & 1 & & 2 & 2 & 3 & & 4 & 4 & 5 & 6 & 7 & $\sum$ & $\%$ \\
\hline Sorex araneus & & 13 & & & & & & 3 & 4 & 7 & $2-$ & 27 & 0.44 \\
\hline Buteo buteo & & 6 & & & & & & & & 1 & & 7 & 0.11 \\
\hline Coccothraustes coccothr. & $1+$ & 8 & & & & & & 1 & 2 & & $1-$ & 11 & 0.18 \\
\hline Elaphe longissima & $1+$ & 5 & & & & & & & & & & 5 & 0.08 \\
\hline Hymenoptera sp. & $1+$ & 8 & & & & & & & & & & 8 & 0.13 \\
\hline Apodemus flavicollis & & 36 & & 7 & & & & 3 & 11 & $1-$ & 3- & 57 & 0.93 \\
\hline Talpa europaea & $2+$ & 113 & $1+$ & 31 & $1+$ & 38 & $1+$ & 25 & 14 & $3-$ & 115 & 337 & 5.49 \\
\hline Turdus philomelos & $2+$ & 17 & $2+$ & 17 & & & & 1 & $1-$ & & $2-$ & 35 & 0.57 \\
\hline Garrulus glandarius & $2+$ & 16 & $1+$ & 6 & & 2 & & & $1-$ & & $2-$ & 24 & 0.39 \\
\hline Bufo bufo & $1+$ & 11 & $1+$ & 7 & & & & 1 & 5 & 2 & $2-$ & 26 & 0.42 \\
\hline Sturnus vulgaris & $1+$ & 7 & & & $1+$ & 7 & & & & & $1-$ & 14 & 0.23 \\
\hline Myodes glareolus & $2+$ & 52 & & 11 & & 5 & $1+$ & 11 & 12 & $1-$ & 4- & 95 & 1.55 \\
\hline Apodemus sylvaticus & & 1 & $1+$ & 7 & & 4 & & 3 & 10 & 1 & $2-$ & 26 & 0.42 \\
\hline Arvicola terrestris & & 7 & $1+$ & 7 & & & & 2 & 3 & & 10 & 29 & 0.47 \\
\hline Turdus merula & & & $1+$ & 6 & & 4 & & & 3 & 2 & $1-$ & 15 & 0.24 \\
\hline Lacerta agilis & & 2 & $1+$ & 15 & & & & 3 & $1-$ & $1-$ & 30 & 54 & 0.88 \\
\hline Anguis fragilis & & 8 & $3+$ & 39 & & & & 2 & $2-$ & $1-$ & 26 & 76 & 1.24 \\
\hline Lepus europaeus & $1-$ & 4 & $1+$ & 21 & $1+$ & 11 & & 6 & 16 & 6 & 33 & 97 & 1.58 \\
\hline Alauda arvensis & & & $1+$ & 8 & $1+$ & 7 & & & & & $1-$ & 15 & 0.24 \\
\hline Cricetus cricetus & $2-$ & 0 & $1-$ & 0 & $3+$ & 56 & & & $2-$ & $2-$ & 57 & 117 & 1.91 \\
\hline Emberiza citrinella & & 1 & & & $1+$ & 6 & & & 2 & 1 & $1-$ & 10 & 0.16 \\
\hline Columba livia dom. & & 5 & & & $1+$ & 9 & & & 1 & 5 & $2-$ & 20 & 0.33 \\
\hline Coleoptera sp. & & 45 & 3- & 0 & & 19 & $3+$ & 79 & $1+137$ & $1+69$ & $5-$ & 350 & 5.70 \\
\hline Gryllotalpa gryllota & 1- & 0 & $1-$ & 0 & & & & & $2+\quad 58$ & $1-\quad 0$ & $2-$ & 65 & 1.06 \\
\hline Orthoptera sp. & $2-$ & 4 & $2-$ & 0 & $2-$ & 0 & $2-$ & 0 & $2+221$ & $2-$ & 12 & 240 & 3.91 \\
\hline Gallus gallus dom. & & & & & & 2 & & & 3 & $1+7$ & 4 & 16 & 0.26 \\
\hline Phasianus colchicus & & & & & & & & 1 & $1-$ & $2+\quad 27$ & $1-\quad 11$ & 40 & 0.65 \\
\hline Lepidoptera sp. & $3-$ & 0 & 3- & 0 & $2-$ & 0 & $2-$ & 0 & 4- & $2+87$ & $1+172$ & 259 & 4.22 \\
\hline Microtus arvalis & & 161 & $1-$ & 156 & $2-$ & 61 & $1-$ & 48 & 587 & 323 & $1+1896$ & 3232 & 52.66 \\
\hline Terricola subterraneus & & 5 & & 1 & & & & 2 & 2 & & $1-\quad 0$ & 10 & 0.16 \\
\hline Perdix perdix & & & & 1 & & 2 & & & 5 & 1 & 18 & 27 & $0 . \overline{44}$ \\
\hline Lacerta vivipara & & & & 5 & & & & & 3 & & 10 & 18 & 0.29 \\
\hline Oryctolagus cuniculus & & & & & & 3 & & & & 1 & 12 & 16 & 0.26 \\
\hline Mustela nivalis & & 1 & & 1 & & & & 1 & 1 & 1 & 10 & 15 & 0.24 \\
\hline Capreolus capreolus & & & & 2 & & 4 & & & & & 4 & 10 & 0.16 \\
\hline Rattus norvegicus & & & & 3 & & & & & 1 & 2 & 3 & 9 & 0.15 \\
\hline Sciurus vulgaris & & 2 & & 1 & & & & 1 & 1 & & 4 & 9 & 0.15 \\
\hline Natrix natrix & & 2 & & & & & & & & & 6 & 8 & 0.13 \\
\hline Pisces sp. & & & & & & & & & 1 & 1 & 6 & 8 & 0.13 \\
\hline Rana temporaria & & 2 & & 4 & & & & & & & & 6 & 0.10 \\
\hline Corvus corone & & & & 3 & & & & & 1 & 2 & & 6 & 0.10 \\
\hline Fringilla coelebs & & 2 & & & & 3 & & 1 & & & & 6 & 0.10 \\
\hline Microtus agrestis & & 1 & & & & 1 & & & 3 & 1 & & 6 & 0.10 \\
\hline Neomys fodiens & & 2 & & & & & & & 1 & 3 & & 6 & 0.10 \\
\hline Mus cf. musculus & & 1 & & & & & & & 4 & & & 5 & 0.08 \\
\hline Carduelis carduelis & & & & & & & & 1 & 4 & & & 5 & 0.08 \\
\hline Mammalia & & 406 & & 266 & & 215 & $1-$ & 105 & 1- 735 & 376 & 2248 & 4351 & 70.89 \\
\hline Aves & $1+$ & 101 & $1+$ & 48 & $2+$ & 72 & & 17 & 79 & $1+87$ & 2- $\quad 39$ & 443 & 7.22 \\
\hline Amphibia, Reptilia, Pisces & & 41 & $1+$ & 70 & $2-$ & 2 & $1-$ & 6 & $2-\quad 26$ & 3- 5 & $1+264$ & 414 & 6.74 \\
\hline Evertebrata & 1- & 58 & 4- & 0 & $1-$ & 19 & $1+$ & 79 & $1+418$ & $1+160$ & 1- 196 & 930 & 15.15 \\
\hline $\bar{\Sigma}$ & & 606 & & 384 & & 308 & & 207 & 1258 & 628 & 2747 & 6138 & 100.00 \\
\hline $\begin{array}{l}\mathrm{H}^{\prime} \text { diversity index / } \\
\text { index diverzity }\end{array}$ & & 2.74 & & 2.38 & & 2.65 & & 2.06 & 2.07 & 1.93 & 1.36 & 2.31 & \\
\hline
\end{tabular}


Tab. 5. Comparison of $B$.buteo food composition with diet of $A$. gentilis and $A$. pomarina in Central Slovakia

Tab. 5. Porovnanie potravy Buteo buteo s potravou Accipiter gentilis a Aquila pomarina na strednom Slovensku

\begin{tabular}{|c|c|c|c|c|c|c|c|c|}
\hline \multirow{2}{*}{$\begin{array}{l}\text { species / druhy } \\
\text { Microtus arvalis }\end{array}$} & \multicolumn{2}{|c|}{ Apom } & \multicolumn{2}{|c|}{ Bbut } & \multicolumn{2}{|c|}{ Agen } & \multirow{2}{*}{$\frac{\Sigma}{559}$} & \multirow{2}{*}{$\begin{array}{r}\% \\
37.62\end{array}$} \\
\hline & $1+$ & 394 & $1-$ & 161 & 4- & 4 & & \\
\hline Microtus agrestis & $1+$ & 40 & $2-$ & 1 & $1-$ & 0 & 41 & 2.76 \\
\hline Arvicola terrestris & $1+$ & 57 & $2-$ & 7 & $2-$ & 0 & 64 & 4.31 \\
\hline Mustela nivalis & $1+$ & 11 & & 1 & & & 12 & 0.81 \\
\hline Rana temporaria & $1+$ & 13 & & 2 & & & 15 & 1.01 \\
\hline Talpa europaea & $2-$ & 16 & $1+$ & 113 & 3- & 0 & 129 & 8.68 \\
\hline Sorex araneus & $1-$ & 0 & $1+$ & 13 & & & 13 & 0.87 \\
\hline Apodemus flavicollis & $1-$ & 9 & $1+$ & 36 & & 5 & 50 & 3.36 \\
\hline Myodes glareolus & 3- & 0 & $1+$ & 52 & $1-$ & 1 & 53 & 3.57 \\
\hline Bufo bufo & $1-$ & 0 & $1+$ & 11 & & & 11 & 0.74 \\
\hline Coleoptera sp. & $2-$ & 3 & $1+$ & 45 & $2-$ & 0 & 48 & 3.23 \\
\hline Hymenoptera sp. & & & $1+$ & 8 & & & 8 & 0.54 \\
\hline Columba livia dom. & 3- & 4 & $3-$ & 5 & $2+$ & 94 & 103 & 6.93 \\
\hline Garrulus glandarius & $2-$ & 1 & & 16 & $2+$ & 31 & 48 & 3.23 \\
\hline Buteo buteo & $1-$ & 0 & & 6 & $1+$ & 8 & 14 & 0.94 \\
\hline Phasianus colchicus & & 1 & & & $1+$ & 6 & 7 & 0.47 \\
\hline Streptopelia decaocto & $1-$ & 0 & $1-$ & 0 & $1+$ & 13 & 13 & 0.87 \\
\hline Dendrocopos major & $1-$ & 0 & $1-$ & 0 & $1+$ & 12 & 12 & 0.81 \\
\hline Turdus merula & & 1 & $1-$ & 0 & $1+$ & 11 & 12 & 0.81 \\
\hline Turdus philomelos & $2-$ & 1 & & 17 & $1+$ & 20 & 38 & 2.56 \\
\hline Sciurus vulgaris & $1-$ & 0 & & 2 & $1+$ & 9 & 11 & 0.74 \\
\hline Coccothraustes coccothr. & $1-$ & $\underline{0}$ & - & 8 & - & 6 & 14 & $0.9 \underline{4}$ \\
\hline Lepus europaeus & & 5 & & 4 & & 2 & 11 & $0 . \overline{4}$ \\
\hline Anguis fragilis & & 3 & & 8 & & & 11 & 0.74 \\
\hline Sturnus vulgaris & & 1 & & 7 & & 2 & 10 & 0.67 \\
\hline Columba palumbus & & 2 & & 3 & & 3 & 8 & 0.54 \\
\hline Perdix perdix & & 2 & & & & 5 & 7 & 0.47 \\
\hline Strix aluco & & & & 1 & & 5 & 6 & 0.40 \\
\hline Columba oenas & & & & 1 & & 4 & 5 & 0.34 \\
\hline Terricola subterraneus & & & & 5 & & & 5 & 0.34 \\
\hline Elaphe longissima & & & & 5 & & & 5 & 0.34 \\
\hline Mammalia & $1+$ & 541 & & 406 & 3- & 22 & 969 & 65.21 \\
\hline Aves & 2- & 34 & 1- & 101 & $2+$ & 260 & 395 & 26.58 \\
\hline Amphibia, Reptilia & & 19 & $1+$ & 41 & $2-$ & 0 & 60 & 4.04 \\
\hline Evertebrata & 2- & 4 & $1+$ & 58 & 2- & 0 & 62 & 4.17 \\
\hline$\Sigma$ & & 598 & & 606 & & 282 & 1486 & 100.00 \\
\hline $\begin{array}{l}\mathrm{H}^{\prime} \text { diversity index / } \\
\text { index diverzity }\end{array}$ & & 1.55 & & 2.74 & & 2.72 & 2.81 & \\
\hline
\end{tabular}

$\overline{A p o m ~-~ A q u i l a ~ p o m a r i n a ~(D r a v e c k y ́ ~ e t ~ a l . ~ 2008, ~ c e n t r a l ~ S l o v a k i a ~ / ~ s t r e d n e ́ ~ S l o v e n s k o), ~ B b u t ~-~ B u t e o ~ b u t e o ~(o w n ~ d a t a ~ / ~ v l a s t n e ́ ~ u ́ d a j e), ~}$ Agen - Accipiter gentilis (Šotnár 2000)

A study of the diet of two similar sized birds of prey; Accipiter gentilis (Šotnár 2000) and Aquila pomarina (Dravecký et al. 2008) carried out in Upper Nitra region were studied in the Upper Nitra region: compare their diets (Tab. 5). In A. pomarina greater proportion of the diet comprises $M$. arvalis and several other non-sylvatic vole species: $M$. agrestis and $A$. terrestris. The most frequently hunted frog species include $R$. temporaria, whereas $B$. buteo hunts $B$. bufo more frequently. A. gentilis is markedly specialized to hunting birds ( $92 \%$ of the diet), few mammals killed include mainly Sciurus vulgaris. Block comparison of species comprising the majority of the diet shown in Tab. 5 reveals that the food niches of these 3 larger bird of prey species have a minimal overlap. 


\section{References}

Cramp S \& Simmons KEL (eds) 1980: The Birds of the Western Palearctic, Vol. II., Hawks to Busards. Oxford Univ Press, Oxford, London \& New York. 696.

Dravecký M, Danko Š, Obuch J, Kicko J, Maderič B, Karaska D, Vrána J, Šreibr O, Šotnár K, Vrlík P \& Bohačík L 2008: Diet of the Lesser Spotted Eagle (Aquila pomarina) in Slovakia. Slovak Rapt J 2: 1-18.

Glutz von Blotzheim UN, Bauer KM \& Bezzel E 1989: Handbuch der Vögel Mitteleuropas. Bd. 4 Falconiformes [2., durchgesehene Auflage]. Aula Verlag, Wiesbaden, 943.

Horák P \& Matušík H 2000: Ještě k potravě káně lesní [Notes on the diet of Common Buzzard]. Crex 16: 33-37. [In Czech with English summary]

Mebs T 1964: Zur Biologie und Populationsdynamik des Mäusebussards (Buteo buteo). J Ornithol 105(3): 247-306.

Obuch J 2001: Using marked differences from the mean (MDFM) method for evaluation of contingency tables. Buteo 12: 37-46.

Sabadoš K \& Sládek J 1968: Príspevok k poznaniu potravy dravcov a sov v bažantnici počas mimoriadne tuhej zimy 1962-1963 [Beitrag zur Kenntnis der Nahrung der Greifvögel und der Eulen in einer Fasanerie während des aussergewöhnlich strengen Winters 1962-1963]. Biológia 23 (5): 363-369. [In Slovak with German summary]

Shannon CE \& Weaver W 1949: The mathematical theory of communication. Univ Illinois Press, Urbana, 125.

Sládek J 1961: Príspevok k poznaniu potravnej ekológie myšiaka lesného Buteo buteo (L.) [Beitrag zur Kenntnis der Nahrungsökologie des Mäusebussards Buteo buteo L.]. Zool listy, 10(1): 331-343. [In Slovak with German summary]

Sládek J 1966: Poznanie zákonitostí potravnej ekológie ako základ hodnotenia hospodárskeho významu myšiaka lesného (Buteo buteo) [Erkenntnis der Gesetzmässigkeiten der Ernährungsökologie als Grundlage der wirtschaftlichen Bedeutung des Mäusebussards (Buteo buteo)]. Českoslov Ochr Prírody 12: 93-103. [In Slovak with German and Russian summaries]
Ševčík J 1980: Potrava káně lesní (Buteo buteo), káně rousné (Buteo lagopus) a jestrába lesního (Accipiter gentilis) $\mathrm{v}$ oblastech $\mathrm{s}$ intenzivním chovem bažantů [Die Ernährung des Mäusebussards (Buteo buteo), Rauhfussbussards (Buteo lagopus) und Habichts (Accipiter gentilis) in der Gegend mit einer Fasanszucht]. Sylvia 20: 35-43. [In Czech with German summary]

Šotnár K. 2000: Príspevok k hniezdnej biológii a potravnej ekológii jastraba vel'kého (Accipiter gentilis) na hornom Ponitrí [A contribution to the breeding biology and feeding ecology of the Goshawk (Acipiter gentilis) in the Horné Ponitrie Region]. Buteo 11: 43-50. [In Slovak with English summary]

Šotnár K 2003: Poznatky o hniezdnej populácii myšiaka hôrneho (Buteo buteo) [Knowledge on breeding population of Common Buzzard (Buteo buteo)]. Vtáčie správy 11(1): 17. [In Slovak]

Šotnár K 2007: Dva roky spod hniezd myšiakov hôrnych [Two years below the nests of Common Buzzard]. Dravce a sovy 3(1): 18-19. [In Slovak with English summary]

Šotnár K \& Topercer J 2009: Estimating density, population size and dynamics of Common Buzzard (Buteo buteo) in a West Carpathian region by a new method. Slovak Rapt J 3: 1-12.

Toufar J 1958: Příspěvek k poznání potravy mladých kání lesních (Buteo buteo) podle zbytků kořisti na hnízdech [Zur Kenntnis der Nahrung nestjunger Mäusebussarde (Buteo buteo)]. Sylvia 15: 67-76. [In Czech with German and Russian summaries]

Uttendörfer O 1939: Die Ernährung der deutschen Raubvögel und Eulen und ihre Bedeutung in der heimischen Natur. Verlag J Neumann, Neudamm, 412.

Voříšek P, Krištín A, Obuch J \& Votýpka J 1997: Potrava káně lesní v České republice a její význam pro myslivost [Diet of Common Buzzard in the Czech Republic and its importance for gamekeeping]. Buteo 9: 57-68. [In Czech with English summary]

Wuttky K 1963: Beutetier-Funde in Greifvögelhorsten des Hakel. Beitr Vogelkd 93(1/2, 3): 140-171. 